\title{
Orientational Distribution of Collagen Fibers in the Horizontal Plane of Human Adult Calcaneus
}

\author{
By Shigeyoshi OSAKI,,$^{1, *}$ Kazuo OHUCHI, ${ }^{2}$ Shin-ichi KIKUCHI, ${ }^{2}$ \\ Yoshiyuki ToHNO, ${ }^{3}$ Setsuko ToHNO, ${ }^{3}$ and Yoshinori TAKAKURA ${ }^{4}$
}

Human right calcaneus bone consisting of hydroxyapatite and collagen fibers was cut in the horizontal plane, which was parallel to the long axis of the calcaneus bone and perpendicular to the human lower limb, into slice samples approximately $1.5 \mathrm{~mm}$ thick. The angular dependence of transmitted microwave intensity at $12 \mathrm{GHz}$ was measured for each slice sample using Osaki's microwave method. It was found that the collagen fibers were mainly aligned in the direction of the long axis of the calcaneus in the region between the heel end and the middle and only in the upper and plantar layers near the anterior, but randomly aligned in the central part of the calcaneus near the anterior. The orientation of collagen fibers changed drastically near the neck, defined as the position where the posterior face of the talus contacts the calcaneus. It is concluded that the orientational distribution of collagen fibers in the horizontal plane of the calcaneus provides the strong resistance against the large stress when the mechanical stress is applied to the neck of the calcaneus from the lower limb.

KEY WORDS: Human Calcaneus / Collagen Fiber / Orientation / Horizontal Plane / Microwave Method /

The fracture of human bones, such as the calcaneus, is often affected by factors such as impact strength and osteoporosis. It is important to study the mechanical properties of human bones and to analyze fractured bones from the viewpoint of orthopedic biomechanics. However, it is relatively difficult to use mechanical methods because of difficulties in preparing sliced bone samples for direct measurement of mechanical anisotropy. For such purposes, alternative methods have been used, including ultrasound velocity, ${ }^{1}$ neutron diffraction, ${ }^{2}$ X-ray diffraction ${ }^{3}$ and optical, ${ }^{4}$ photoelastic $^{5}$ methods to estimate the physical anisotropy of human and animal bones. $^{6-10}$

It is necessary to determine the orientation of collagen fibers in bones consisting of hydroxyapatite and collagen in order to discuss mechanical anisotropy at the molecular level. However, it is difficult to determine the fiber orientation of collagen in bones using methods ${ }^{1-3}$ such as ultrasound velocity, neutron diffraction and X-ray diffraction. And, circular polarized light has been used to evaluate optical anisotropy in thin samples of ovine calcaneus bone $e^{4,11}$ and osteonal bone. ${ }^{8}$ However, it is also difficult to determine the orientational distribution of collagen fibers in bulk bones using optical methods, ${ }^{4,8,11}$ which provide information related to the refractive index coming from the electron cloud. Thus, a more suitable method is needed to determine the orientation of collagen fibers in thick and opaque bones from both the fundamental and clinical points of view.

Previously, Osaki's microwave method for determining molecular and fiber orientation was established, ${ }^{12-17}$ which is effective within $10 \mathrm{~s}$ for thick and opaque samples, and this technique has been applied to human blood vessels, ${ }^{18}$ and calf $^{19,20}$ and cobra skin. ${ }^{21-23}$ The microwave method clarified that the orientational distribution of collagen fibers is closely related to mechanical anisotropy.

Here, the authors attempted to measure fiber orientation in samples cut from human calcaneus bone in three different planes (frontal, sagittal and horizontal) using Osaki's microwave method. In the preceding papers, ${ }^{24,25}$ fiber orientation of human calcaneus bone was measured in two planes (frontal and sagittal). However, fiber orientation was not determined in the horizontal plane.

The present paper thus describes the orientational distribution of collagen fibers in slice samples cut in the horizontal plane, parallel to the long axis of the human right calcaneus bone and perpendicular to the human lower limb, and provides information on the mechanical resistance to stresses applied to the calcaneus bone.

\section{EXPERIMENTAL}

\section{Preparation of Samples Cut in Horizontal Plane From Calcaneus Bone}

A sample from a 71-year-old woman was fixed in a solution of $36 \%$ ethanol, $13 \%$ glycerin, $6 \%$ phenol and $6 \%$ formalin, and was used for anatomical training by medical students. We subsequently obtained the right calcaneus, which was healthy bone. The shape of the calcaneus bone was roughly similar to a cylindrical rod with a diameter of about $3.5 \mathrm{~cm}$ between the heel end and middle, but was not cylindrical at the anterior and facing the toe (Figure 1A). Here, the neck is defined as the point where the posterior face of the talus contacts the

\footnotetext{
${ }^{1}$ Department of Chemistry, Nara Medical University, Kashihara 634-8521, Japan

${ }^{2}$ Department of Orthopaedics, Fukushima Medical University, Fukushima 960-1295, Japan

${ }^{3}$ Department of Anatomy, Nara Medical University, Kashihara 634-8521, Japan

${ }^{4}$ Department of Orthopaedics, Nara Medical University, Kashihara 634-8521, Japan

*To whom correspondence should be addressed (Tel/Fax: +81-744-29-8810, E-mail: s-osaki@ naramed-u.ac.jp).
} 


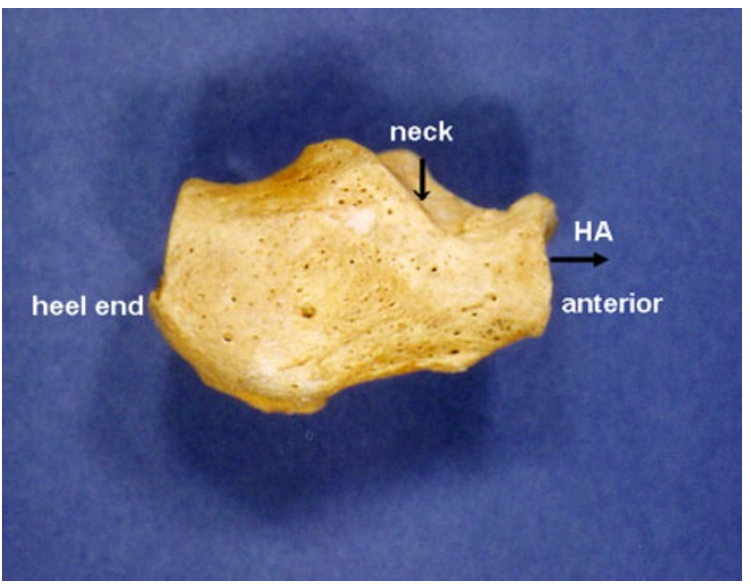

(A)

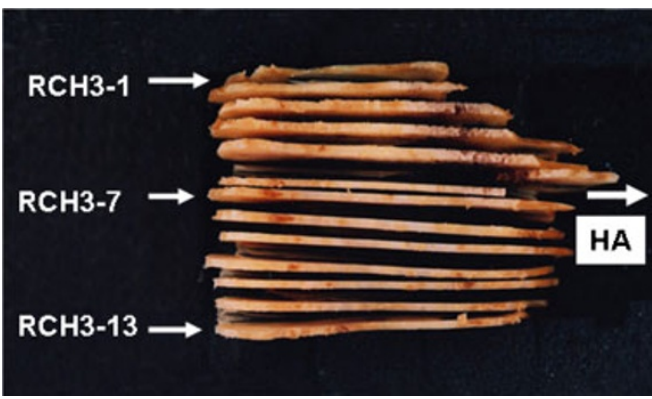

(B)

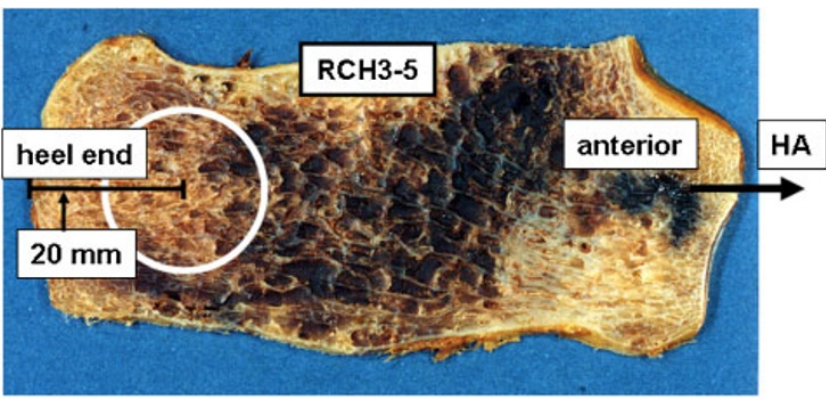

(C)

Figure 1. A, Side view of human right calcaneus bone. $\mathrm{HA}$ is defined as the direction from the heel to anterior ends. B, Slices ( $\mathrm{RCH} 3-1$ to $\mathrm{RCH} 3-13)$ cut parallel to the horizontal plane of the right calcaneus bone. C, Slices (RCH3-5) cut in the horizontal plane of right calcaneus bone. Microwave measurements were carried out $20 \mathrm{~mm}$ from the heel end. Circle indicates the effective area of microwaves irradiation.

calcaneus. In addition, HA is defined as the direction from the heel to the anterior end of the calcaneus bone.

The right calcaneus bone was cut in the horizontal plane parallel to the long axis of the right calcaneus bone and perpendicular to the human lower limb, into 13 sheets of approximately $1.5-\mathrm{mm}$ thickness (see Figure 1B) using an MC120 cutter (Maruto Co., Japan). The sliced sheets were dried by air flow at $50{ }^{\circ} \mathrm{C}$ for $1 \mathrm{~d}$, kept at room temperature for more than $5 \mathrm{~d}$, and samples were then prepared. Here, the sheets were designated as specimens RCH3-1 to RCH3-13,

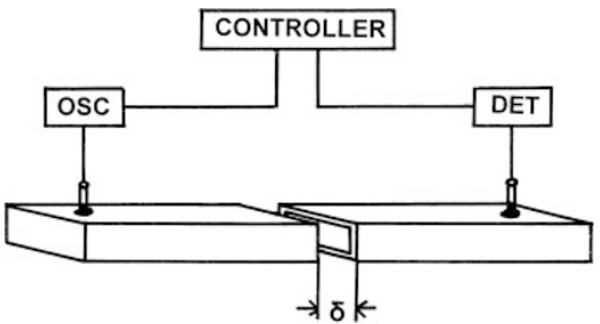

(A)

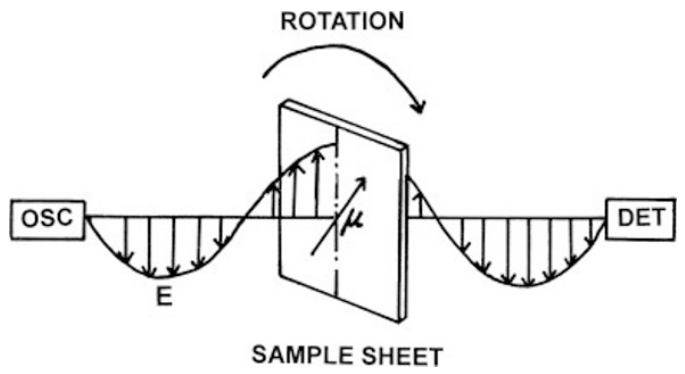

(B)

Figure 2. A, Cavity resonator system consisting of a pair of rectangular waveguides with narrow gap $\delta$. The cavity resonator is connected with an oscillator (OSC) and a detector (DET). The sample sheet set in the gap is rotated by a controller (CONTROLLER). B, When the sample sheet is set at the abdomen of the electric field $(E)$ of microwaves, both dipole moments $(\mu)$ in the sample and the electric field have a maximum correlation so that the fiber orientation is detected most effectively.

based on positions from the upper to the plantar side of the right calcaneus bone (Figure 1B) and were used for soft X-ray photos, optical photos and microwave measurements. The red circle of $16 \mathrm{~mm}$ in diameter shown in the plane of RCH3-5 (Figure 1C) represents the effective area of microwave irradiation.

The chemical content of the calcaneus bone, other than water and lipids, was determined to be about $35 \%$ organic material such as collagen fibers, and about $65 \%$ inorganic hydroxyapatite, consisting of calcium phosphate, calcium carbonate, and magnesium phosphate.

\section{Microwave Measurements}

Figure 2A shows the cavity resonator system, which consists of a pair of rectangular waveguides separated by a narrow gap whose width is here denoted by $\delta$. One of waveguides is connected to an oscillator (OSC) of a cavity type and the other to a detector (DET) of a GaAs. The dimension of the waveguides WRJ-15 are $15.8 \mathrm{~mm}$ wide and $7.9 \mathrm{~mm}$ high. Each slice sample was inserted into the narrow gap of the cavity resonator system, and was rotated for $6.0 \mathrm{~s}$ around the axis central to the sample plane by a controller (CONTROLLER) (Figure 2A and 2B). When the sample sheet is set at the center of the gap where the abdomen of the electric field (E) of microwaves exists, both dipole moments $(\mu)$ of molecular chains in the sample and the electric field have a maximum correlation so that the fiber orientation should be detected most effectively. 
Microwaves (12 GHz) were transmitted in this sample plane, and the microwave intensity was measured at different rotation angles within $6.0 \mathrm{~s}^{12,15,23,25}$ Such measurements were carried out at different positions by successively shifting the position of the sample at intervals of $5 \mathrm{~mm}$ in the HA direction (see Figure 1C). As the transmitted microwaves indicate the interaction between polarized microwaves and dipole moments, ${ }^{12-14}$ the angular dependence of the transmitted microwave intensity reflects the distribution of fiber orientation, giving the orientation angle (ANGLE) and degree of fiber orientation (DFO). ${ }^{17,19}$ For example, the angular dependence for samples with a random orientation of fibers or a preferred orientation of fibers gives a circular pattern or a pattern like an ovaloid, respectively. ${ }^{15,17}$ Thus, Osaki's microwave method gives fiber orientation, which is independent of mineral intensity of the calcaneus bone and mainly reflects collagen fibers in the calcaneus bone. ${ }^{24,25}$ The direction giving the minimum transmitted microwave intensity was designated as ANGLE, ${ }^{15,17}$ corresponding to the angle between the main axis of collagen-fiber chains and the HA direction. The maximal-tominimal ratio of transmitted microwave intensity was also defined as DFO, which increases by increasing the preferential alignment of fibers. ${ }^{20,24}$ As a DFO of 1.0 reflects random orientation, a value of (DFO-1.0), which is zero or positive, reflects the net degree of orientation.

The resonance curve before and after insertion of a sample in the cavity resonator system was thus expressed in terms of transmitted microwave intensity I. ${ }^{12,15}$

$$
\mathrm{I}(\theta)=\mathrm{I}_{\mathrm{i} 0}(\theta) /\left\{1+\mathrm{Q}_{\mathrm{i}}{ }^{2}(\theta)\left[\mathrm{f}_{\mathrm{i} 0}(\theta) / \mathrm{f}-\mathrm{f} / \mathrm{f}_{\mathrm{i} 0}(\theta)\right]^{2}\right\}
$$

where, $I, I_{i 0}$ and $Q_{i}(i=1,2)$ are transmitted microwave intensities at a given frequency $f$, its value at resonance frequency $f_{i 0}$, and the ratio of resonance frequency to half the width of $\Delta \mathrm{f}$ in the resonance curve, respectively, all at rotation angle $\theta$. Subscripts 1 and 2 indicate values before and after insertion of the sample.

In the actual experiments, the angular dependence of transmitted microwave intensity in the resonance curve was measured at a fixed frequency, where the intensity was half the peak intensity, higher than the resonance frequency. ${ }^{15,17}$ Errors in the values of ANGLE and DFO measured by Osaki's microwave method were within $\pm 1^{\circ}$ and $\pm 5 \%$, respectively. Errors in ANGLE depend on adjustment of the sample axis in the HA direction, while errors in DFO also depend on variations in sample thickness.

\section{Soft X-ray Photographs}

Each slice of the right calcaneus was set in a Softex-E40, and X-ray photographs were developed after $20 \mathrm{~s}$ of irradiation with soft X-rays. The fine structure of trabeculae was then examined.

\section{RESULTS}

Fine Structure of Calcaneus Bone Using Soft X-rays

Figure 3 shows soft X-ray photographs for four different

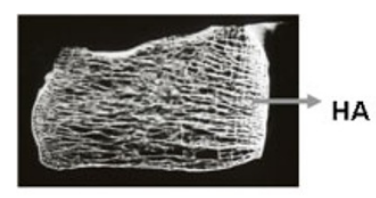

$\mathrm{RCH} 3-2$

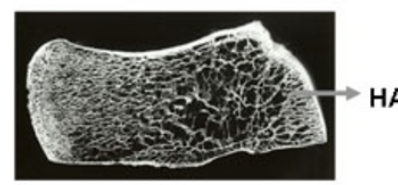

$\mathrm{RCH} 3-8$

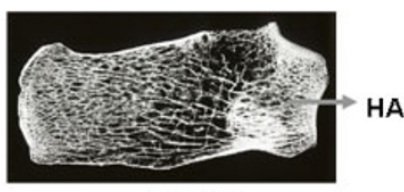

RCH3-5

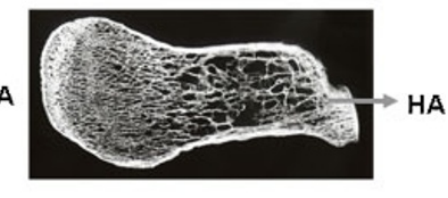

RCH3-12
Figure 3. Soft $X$-ray photographs of four different slices $(\mathrm{RCH} 3-2, \mathrm{RCH} 3-5$ $\mathrm{RCH}-8$, $\mathrm{RCH} 3-12$ ) cut in the horizontal plane of the right calcaneus bone. HA is defined as the direction from the heel to anterior ends.

slices (RCH3-2, RCH3-5, RCH3-8, RCH3-12) selected from 13 samples prepared by cutting the right calcaneus bone in the horizontal plane (see Figure 1B). The distribution of the parts shown in white reflects the fine structures of hydroxyapatite representing trabecular bone. The fine structure varies markedly, depending on the position of the calcaneus slice. The lines are mainly aligned in the HA direction at the heel end and the middle along the long axis of the calcaneus. In addition, an area without lines is observed between the middle and anterior. Such a distribution may be qualitatively related to the orientation of hydroxyapatite crystals in the calcaneus bone. Even if collagen fibers follow the long axis of the calcaneus, however, it will be very difficult to numerically evaluate the orientation of not only the hydroxyapatite crystals but also the collagen fibers using soft X-ray photos. A previous paper ${ }^{24}$ demonstrated that Osaki's microwave method numerically determines the orientation of collagen fibers in the calcaneus. Thus, we attempted to apply Osaki's microwave method to determine fiber orientation in the horizontal plane of the calcaneus bone.

\section{Fiber Orientation of Collagen Near the Heel End in the Horizontal Plane}

Figure 4 shows the angular dependence of transmitted microwaves $(12 \mathrm{GHz})$ at a distance of $20 \mathrm{~mm}$ from the heel end of four different slice samples ( $\mathrm{RCH} 3-2, \mathrm{RCH} 3-5, \mathrm{RCH} 3-$ 8 , RCH3-12) prepared by cutting the right calcaneus bone in the horizontal plane. ANGLE was defined as the angle deviating from the HA direction, indicating the direction of fiber orientation. DFO was defined as the degree of fiber orientation, indicating a measure of dielectric anisotropy. Each of the four angular dependences in Figure 4 gives a similar pattern, indicating similar anisotropy. ANGLE varied from $-6^{\circ}$ to $5^{\circ}$ in positions from $\mathrm{RCH} 3-2-20$ to $\mathrm{RCH} 3-12-20$. Such small values indicate that the fibers are mainly aligned in a fixed direction. DFO varied from 2.241 to 2.718 at positions from $\mathrm{RCH} 3-2-20$ to $\mathrm{RCH} 3-12-20$. DFO was more than 2.0, indicating strong anisotropy, resulting in marked orientation of 


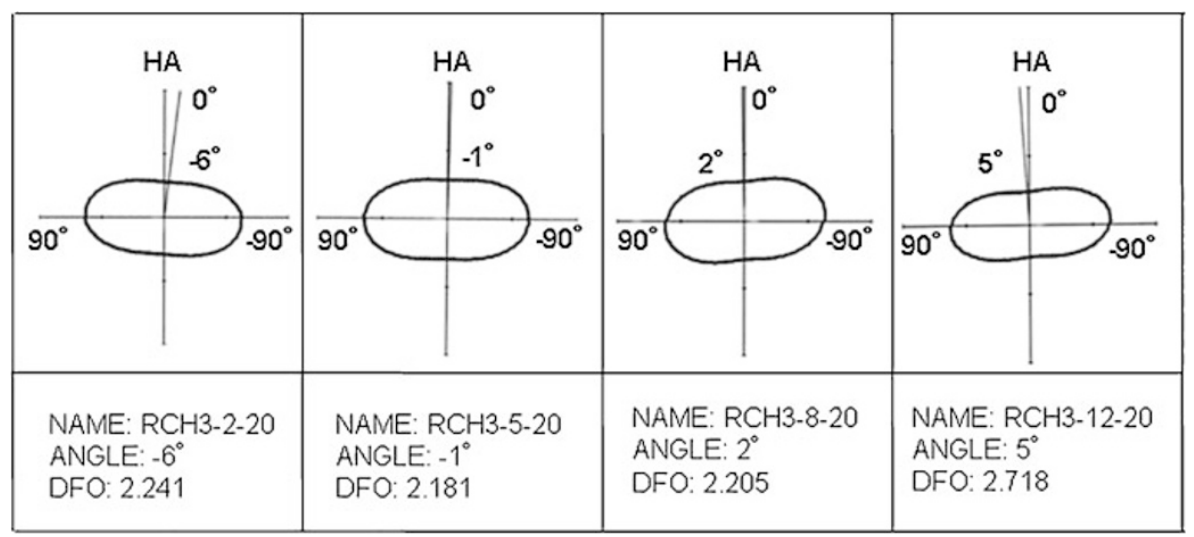

Figure 4. Angular dependence of the transmitted microwave intensity obtained for four different slices $(\mathrm{RCH}-2-20, \mathrm{RCH}-5-20, \mathrm{RCH}-8-20, \mathrm{RCH}-12-20)$ $20 \mathrm{~mm}$ from the heel end of the right calcaneus bone. Orientation angle (ANGLE) is defined as the angle of fibers deviating from the HA direction, and DFO is defined as the degree of fiber orientation.

collagen fibers. ANGLE and DFO determined by Osaki's microwave method are plotted, respectively, in Figure 5A and $5 \mathrm{~B}$ against the position from the upper to the plantar side (RCH3-1 to RCH3-13) at a distance of $20 \mathrm{~mm}$ from the heel end. The deviation in ANGLE from HA is very small, confirming that the fibers are mainly aligned in the HA direction at a distance of $20 \mathrm{~mm}$ from the heel end of the right calcaneus. DFO decreased slightly in the middle of the calcaneus, but increased particularly on the plantar side. This shows that collagen fibers are mainly aligned in the HA direction from the upper to the plantar side, but are markedly aligned near the plantar part.

\section{Fiber Orientation of Collagen between the Heel End and Anterior in the Horizontal Plane}

Next, angular dependence was measured at different positions of the slice sample ( $\mathrm{RCH} 3-5)$ along the long axis of the right calcaneus bone, using Osaki's microwave method. Microwave measurements were carried out at intervals of $5 \mathrm{~mm}$ from the heel end to the anterior along the long axis of the right calcaneus bone, as shown in Figure 6. Each red circle is an area of microwave irradiation. Similarly, microwave measurements were carried out for slice samples ( $\mathrm{RCH} 3-1$ to $\mathrm{RCH} 3-13)$ at intervals of $5 \mathrm{~mm}$ between $5 \mathrm{~mm}$ and $65 \mathrm{~mm}$ from the heel end along the long axis of the right calcaneus bone. ANGLE in the horizontal plane was plotted in Figure 7A against the distance from the heel end in the HA direction for slice samples (RCH3-1 to RCH3-13). ANGLE was small between the heel end and the middle, but was particularly large or small for RCH3-7, RCH3-9 and RCH3-11 near the anterior. Even in the anterior part, ANGLE was small for RCH3-5 and RCH3-13. This suggests that collagen fibers are mainly oriented in the HA direction from the heel end to the middle, but deviate markedly from HA between the middle and the anterior.

DFO was plotted in Figure 7B against the distance from the heel end in the HA direction for samples (RCH3-1, RCH3-3, RCH3-5, RCH3-7, RCH3-9, RCH3-11, RCH3-13) prepared

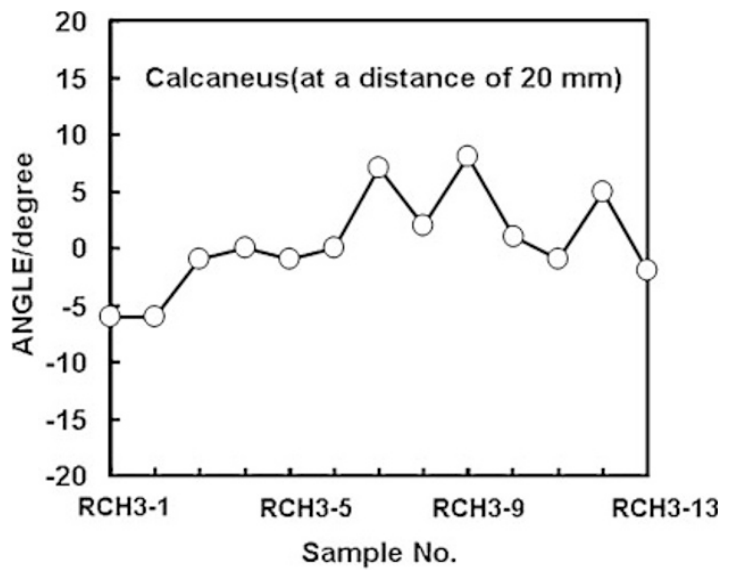

(A)

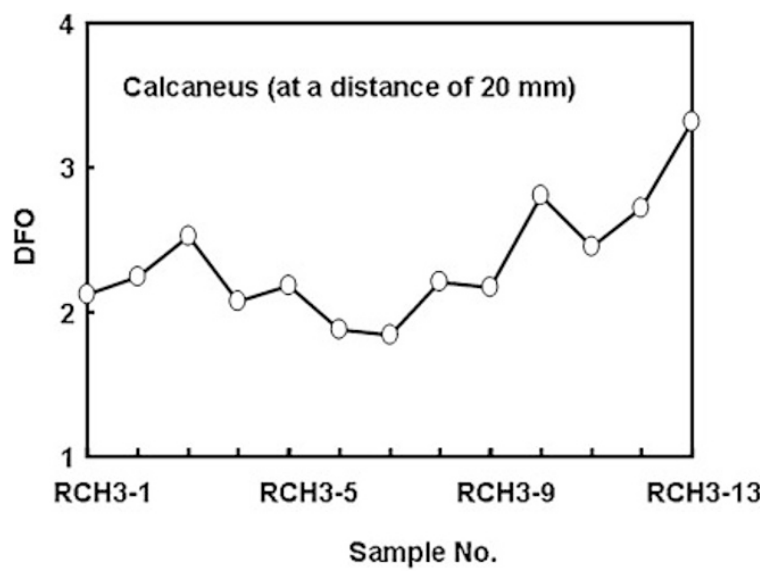

(B)

Figure 5. A, Orientation angle (ANGLE), measured $20 \mathrm{~mm}$ from the heel end of the right calcaneus bone by Osaki's microwave method, was plotted against the position from the upper side to the plantar side of samples (RCH3-1 to RCH3-13). Orientation angle (ANGLE) is defined as the angle of fibers deviating from the HA direction. B, DFO reflecting the degree of fiber orientation, measured $20 \mathrm{~mm}$ from the heel end of the right calcaneus bone by Osaki's microwave method, was plotted against the position from the upper to the plantar side of the samples ( $\mathrm{RCH} 3-1$ to $\mathrm{RCH} 3-13)$. 


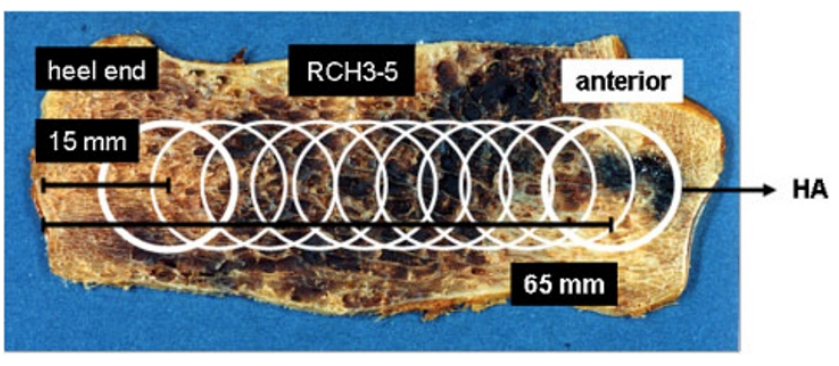

Figure 6. Microwave measurements for slices (ex. $\mathrm{RCH}-5$ ) were carried out at intervals of $5 \mathrm{~mm}$ by shifting the position from the heel end to the anterior along the long axis of the right calcaneus bone. Circles are the effective area of microwave irradiation at different positions.

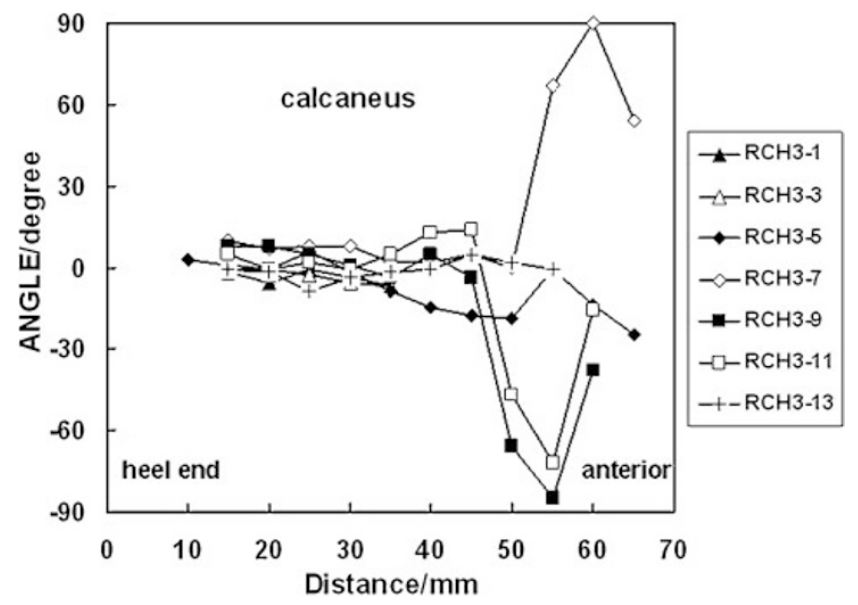

(A)

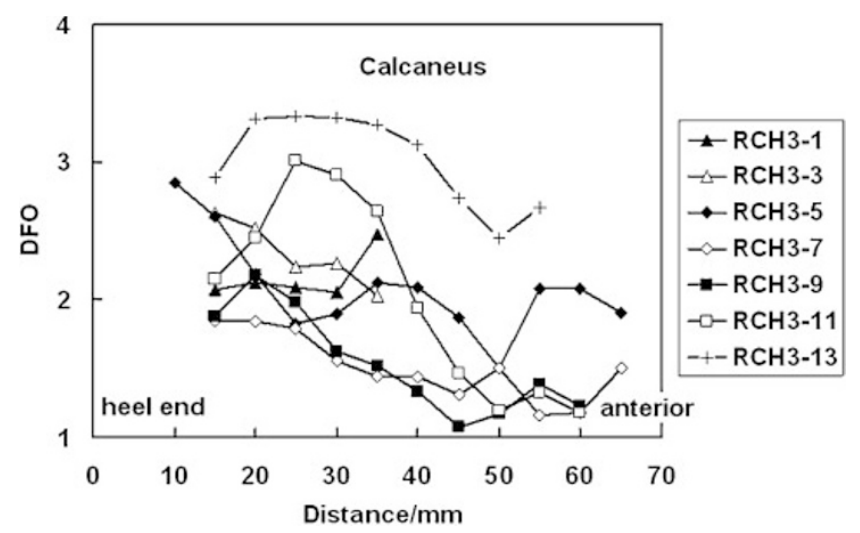

(B)

Figure 7. A, Orientation angle (ANGLE) was plotted against the position of slices (RCH3-1, RCH3-3, RCH3-5, RCH3-7, RCH3-9, RCH3-11, $\mathrm{RCH} 3-13)$ prepared from the right calcaneus bone. Microwave measurements were carried out at intervals of $5 \mathrm{~mm}$ from the heel end to the anterior along the long axis of the right calcaneus bone. $\boldsymbol{\Delta}, \mathrm{RCH} 3-1 ; \triangle, \mathrm{RCH} 3-3 ; \diamond, \mathrm{RCH} 3-5 ; \diamond, \mathrm{RCH} 3-7 ; \mathbf{\square C H}-9$; $\square, \mathrm{RCH} 3-11 ;+, \mathrm{RCH} 3-13$. B, DFO was plotted against the position of slices ( $\mathrm{RCH} 3-1, \mathrm{RCH} 3-3, \mathrm{RCH} 3-5, \mathrm{RCH} 3-7, \mathrm{RCH} 3-9$, $\mathrm{RCH} 3-11, \mathrm{RCH} 3-13$ ) prepared from the right calcaneus bone. Microwave measurements were carried out at intervals of $5 \mathrm{~mm}$ from the heel end to the anterior along the long axis of the right calcaneus bone. $\Delta, \mathrm{RCH} 3-1 ; \triangle, \mathrm{RCH} 3-3 ; \diamond, \mathrm{RCH} 3-5 ; \diamond, \mathrm{RCH} 3-$ 7; $\square$, RCH3-9; $\square, \mathrm{RCH} 3-11 ;+, \mathrm{RCH} 3-13$.
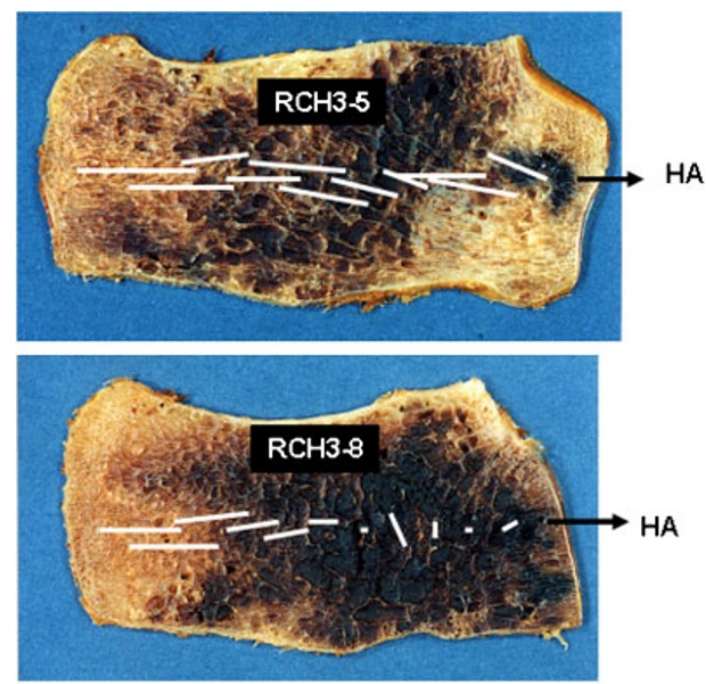

Figure 8. Orientational distribution of collagen fibers determined by Osaki's microwave method for slice samples ( $\mathrm{RCH} 3-5$ and $\mathrm{RCH} 3-8)$ cut in the horizontal plane of the right calcaneus bone. The length and inclination of the line represents the net degree of fiber orientation (DFO-1.0) and the direction of fiber orientation, respectively. HA is defined as the direction from the heel to anterior ends.

from the right calcaneus bone. DFO varied markedly with position. Overall, DFO is large in the heel end and the middle part, meaning that fibers are largely oriented in a fixed direction of the horizontal plane. On the other hand, DFO is relatively small near the anterior part, except for RCH3-5 and RCH3-13 even though ANGLE is large, meaning that collagen fibers are randomly oriented in the anterior part.

The size of the cylindrical calcaneus is small in the anterior part, as compared with that between the heel end and the middle. DFO is large in the anterior part of RCH3-5 and RCH3-13, corresponding to the surface layers of the calcaneus. The small value for DFO indicates that the direction of fiber orientation gives no valuable insight because the collagen fibers are randomly aligned. Here, the limit where fiber orientation changed drastically corresponds to the neck (see Figure 1A), defined as the point where the posterior face of the talus contacts the calcaneus. Thus, collagen fibers are, on average, oriented in the HA direction only in the upper and plantar layers of the anterior even though they are randomly aligned in the central part of the anterior.

The orientational distribution of collagen fibers determined by Osaki's microwave method is visually shown in Figure 8 for RCH3-5 and $\mathrm{RCH} 3-8$ prepared from the right calcaneus bone. The length and inclination of the line represent the net degree of fiber orientation (DFO-1.0) and the direction of fiber orientation, respectively. Overall, the fibers in RCH3-5 and RCH3-13 are mainly oriented in the HA in the total area of the sample plane, but the fibers in the $\mathrm{RCH} 3-8$ corresponding to the central part of the calcaneus are mainly oriented in the HA between the heel end and the middle, and are randomly oriented near the anterior. Thus, the position where DFO changes markedly corresponds to the neck. 


\section{DISCUSSION}

\section{Orientational Distribution of Collagen Fibers in the Frontal and Sagittal Planes}

Previous papers ${ }^{20,21,23}$ have shown that the direction of fiber orientation of collagen determined using Osaki's microwave method correspond to the highest mechanical stress in cow and cobra skins. Similarly, the fiber orientation obtained for calcaneus bone using Osaki's microwave method will give useful information about the mechanical behavior of the whole calcaneus bone, as the mechanical properties are closely related to collagen fiber orientation. ${ }^{20-23}$

The orientation of collagen fibers in the frontal plane of the calcaneus, as shown previously, ${ }^{24}$ suggested that mechanical stress might be applied to the lateral side at the heel of the calcaneus and to the medial side in the middle, and that mechanical stress might be relatively large at the heel and small in the middle of the calcaneus. Another previous paper ${ }^{25}$ showed that ANGLE changed drastically in the sagittal plane near the neck along the long axis of the right calcaneus bone. The results suggest that the orientational distribution of collagen fibers correspond to the dispersion of mechanical stress applied to the neck in two different (frontal and sagittal) directions. The orientational distribution provides a structure effective for avoiding mechanical destruction of the calcaneus bone, including bending around the neck.

If the fiber orientation of collagen is determined in three different perpendicular planes (not only frontal and sagittal, but also horizontal) using Osaki's microwave method, such data will give useful information for estimating the distribution map of mechanical stress in the whole calcaneus bone.

\section{Orientational Distribution of Collagen Fibers in the Hor- izontal Plane}

The main part of the calcaneus bone shows fine structures based on complex bony trabeculae (inner part) with low bone density, while the surface part (upper and plantar) of the calcaneus is covered with a very thin layer of cortical compact bone with high bone density. In other words, the upper and plantar parts are thought to be mechanically strong, while the inner part is thought to be viscoelastic and mechanically weak.

In this paper, the orientation of collagen fibers was determined for samples cut in the horizontal plane of the calcaneus. It was found that the collagen fibers were mainly aligned along the HA (from the heel to the anterior end) direction, while they were randomly aligned between the central and the anterior parts for slice samples. The direction of fiber orientation changed drastically around the neck of the calcaneus, to which most of the body weight should be applied from the lower limb. Our data on orientational distribution will give fundamental information to discuss the bending ascribed to the compressive stress applied to the neck of the calcaneus, although it is very difficult to carry out mechanical measurements of bone.
As shown in Figure 6B, the value of DFO in the upper and plantar parts of the horizontal plane is relatively large compared with that in the inner parts. The results suggest that the upper and plantar parts of the calcaneus have good mechanical strength against the compression stress applied to the neck from the lower limb, while the inner part has a relatively weak structure against this stress. Thus, the calcaneus is best represented by a series model consisting of three layers; the rigid upper part, the relatively soft inner part, and the rigid plantar part. If compression stress is applied mainly to the neck from the lower limb, the strain will be different at the various parts of the calcaneus, with the neck being compressed maximally and deformed markedly. Therefore, the calcaneus as a cylindrical rod may be apt to bend around the neck. On the other hand, the orientational distribution of collagen fibers in the horizontal plane of the calcaneus provides strong resistance to stresses applied to the neck, which supports the results ${ }^{25}$ obtained for the sagittal plane.

The three-dimensional distribution of collagen fiber orientation provides important information regarding the growth of bones and walking styles, and clarifies the various functions of human bone from a clinical point of view. On the other hand, it is known that changes in the mechanical properties ascribed to the osteoporosis often induce fractures in bones, such as the calcaneus. Determination of the three-dimensional orientational distribution of collagen fibers in the healthy calcaneus bone will be useful for providing fundamental information related to osteoporosis from a clinical point of view, and also for proposing a guide to treat the fractured calcaneus.

In the future, it will be necessary to deal with calcaneus bones in subjects with different waking styles and osteoporosis in order to clarify the relationship between mechanical properties and collagen fiber orientation from both fundamental and clinical points of view.

Received: October 15, 2008 Accepted: November 10, 2008 Published: December 26, 2008

\section{REFERENCES}

1. M. A. Greenfield, J. D. Graven, A. Huddleston, M. L. Kehler, D. Wishko, and R. Stern, Radiol., 138, 701 (1981).

2. G. E. Bacon, P. J. Bacon, and R. K. Griffiths, J. Anat., 139, 265 (1984).

3. H. W. Wahner and I. Fogelman, in "The evaluation of osteoporosis," Martin Duntiz, London, 1999.

4. J. M. McMahon, A. Boyde, and T. G. Bromage, Anat. Rec., 242, 147 (1995).

5. H. Gierse, Anat. Embryol., 150, 63 (1976).

6. E. Lozpone, Anat. Anz. Jena., 159, 211 (1985).

7. A. L. Yettram and N. N. Camilleri, J. Biomed. Eng., 15, 46 (1993).

8. R. B. Martin, S. T. Lau, P. V. Masthews, V. A. Gipson, and S. M. Stover, J. Biomech., 29, 1515 (1996).

9. Y. Takano, C. H. Turner, I. Owan, and R. B. Martin, J. Orthopaedic Res., 17, 59 (1999).

10. J. K. Kalmey and C. O. Lovejoy, Bone, 31, 327 (2002).

11. T. G. Bromage, H. M. Goldman, S. C. McFarlin, J. Warshaw, A. Boyde, and C. M. Riggs, Anat. Rec., New. Anat., 274B, 157 (2003). 
12. S. Osaki, Polym. J., 19, 821 (1987).

13. S. Osaki, Tappi J., 70, 105 (1987).

14. S. Osaki, Tappi J., 72, 171 (1989).

15. S. Osaki, J. Appl. Phys., 67, 6513 (1990).

16. S. Osaki, Nature, 347, 132 (1990).

17. S. Osaki, Rev. Sci. Instrum., 68, 2518 (1997).

18. K. Yamamoto, S. Osaki, S. Yamashita, and M.-o. Yamada, Cell. Mol. Biol., 34, 571 (1988).

19. S. Osaki, M.-o. Yamada, A. Takakusu, and K. Murakami, Cell. Mol. Biol., 39, 673 (1993).
20. S. Osaki, Anat. Rec., 254, 147 (1999).

21. S. Osaki and T. Ohashi, Cell. Mol. Biol., 50, 559 (2004).

22. K. Niitsuma, S. Miyagawa, and S. Osaki, Eur. J. Morphol., 42, 193 (2005).

23. K. Niitsuma, S. Miyagawa, and S. Osaki, Polym. J., 39, 181 (2007).

24. S. Osaki, S. Tohno, Y. Tohno, K. Ohuchi, and Y. Takakura, Ana. Rec., 266, 103 (2002).

25. K. Ohuchi, S. Osaki, S. Tohno, Y. Tohno, Y. Takakura, and S.-i. Kikuchi, Cell. Mol. Biol., 49, OL425 (2003). 